\title{
Development of Stent Motor
}

\author{
Shigeki Toyama and Uichi Nishizawa
}

\begin{abstract}
The purpose of this study is to develop a stent motor applicable for medical blood vessel treatment. The stent motor is a movable stent and it has functions of removing plaque and expanding blood vessels. It consists of two parts; ultrasonic receivers and vibration transmitting coil (stator). In this study, the authors have focused in the vibration analysis of the receiver. In the design stage, the authors have evaluated resonant phenomenon using finite element method. When applying it in water, the resonant frequency in the air is shifted due to mass effect of water around the receiver. Frequency response analysis of the receiver shows that extremely high precision of dimensions is required because the resonant frequency in water depends not only size but also the amplitude of the vibration. It is very difficult to design an optimal receivers resonating in water. In order to solve this problem, the authors have tried a new receiver with wide plate receiving ultrasound effectively and requiring no resonant. The authors have succeeded in driving this type of motor in water.
\end{abstract}

Index Terms-Ultrasonic motor, piezoelectric actuator, waterproof actuator and stent.

\section{INTRODUCTION}

Recently coronary heart disease is one of the Japanese three major causes of death in late years. Especially angina and myocardial infarction are serious. These diseases are caused by lack of bloodstream to the myocardium due to stenosis of coronary arteries. A coronary angioplasty is used at the constricted vessel. Usually, a doctor detains a metal pipe (stent) of the form of halftone plate to hold a lumen. However, it becomes constricted again near the stent, and it follows re-operating. If the stent can move back and forth in the blood vessel and expand it, it will prevent restenosis. Therefore the authors have applied the ultrasonic motor technology to the stent to slide in the blood vessel by irradiating a supersonic wave from the outside human body.

\section{DRIVE PRINCIPLE OF STENT MOTOR}

The stent motor consists of a receiver and a coil (stator). The receiver receives energy of the ultrasonic wave vibration in water generated from the outside vibration source. The stator is the coil shape, and transmitting the energy in the form of travelling wave. At the top of amplitude of travelling wave, frictional force (driving force) is generated between stator and blood vessel. The authors have two models; one is based on resonant phenomenon and the other is based on ultrasonic impact.

The motor mechanism is as follows; a vibration source such as Langevin oscillator generates ultrasonic to a human

Manuscript received February 20, 2018; revised April 12, 2018.

Shigeki Toyama and Uichi Nishizawa are with Tokyo University of Agriculture and Technology, Koganei, Tokyo, Japan (e-mail: toyama@cc.tuat.ac.jp,n-uichi@cc.tuat.ac.jp). body from outside. The vibration wave is travelling in water to the motor receiver. The motor receiver starts vibrating with principal vibration mode and inducing traveling wave along the coil (stator). The coil and blood vessel is pressed each other with proper force. The travelling wave generates frictional force at the top of the wave amplitude. It works as driving force to rotate the stent (blood vessel is stationary).

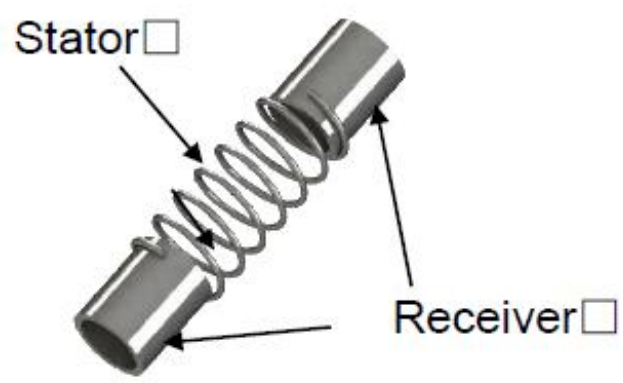

Fig. 1. Schemantic diagram of stent motor.

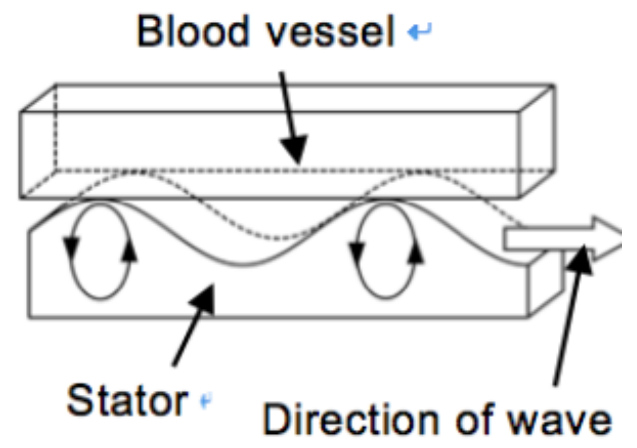

Fig. 2. Elliptic motion of stator.

\section{RESONANT TyPE STENT MOTOR}

\section{A. Characteristics of Lagevin Transducer}

The authors have used bolted Langevin transducer as the vibration source. When applying alternating voltage at the resonant frequency to the Langevin transducer, it generates the large amplitude of vibration. However, the resonant frequency is not fixed and changing with applied voltage (Fig. 3).

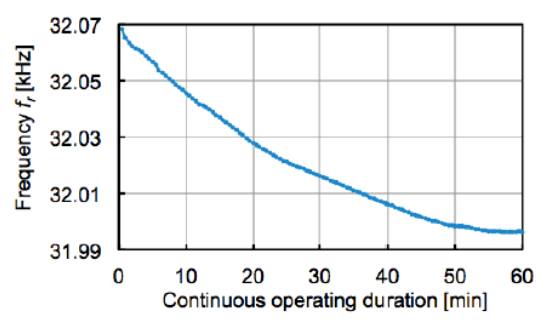

Fig. 3. Relationship between $f_{r}$ and operating duration.

The horn top part of Langevin transducer is set to the $5 \mathrm{~mm}$ 
under the surface of water and the applied voltage is set as 80 $V_{p-p}$. The relationship between drive time and a resonant frequency is shown in Fig.3. A resonant frequency keeps falling from starting to the static state in about 56 minutes. At the static state, the power consumption is $10.32 \mathrm{~W}$ and the resonant frequency $f_{r}$ in is $31,997 \mathrm{~Hz}$. When increasing applied voltage, the resonant frequency is changing as shown in Fig. 4. The resonant frequency is falling with a rise of voltage.

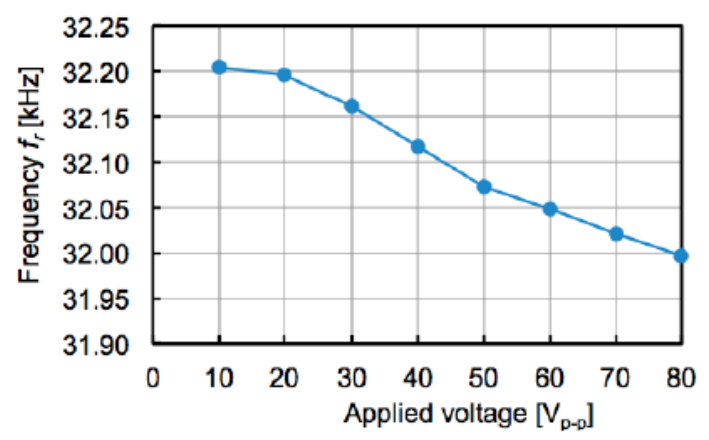

Fig. 4. $f_{r}$ and applied voltage.

The authors have measured vibration by an impedance analyzer and found that resonant frequency is $f_{r}=32.08 \mathrm{kHz}$ and anti-resonance frequency is $f_{a}=33.21 \mathrm{kHz}$. Based on it, the electromechanical coupling coefficient is derived to be 0.2849; the maximum mechanical energy changed from input electric power is $2.94 \mathrm{~W}$.

\section{B. Additional Mass Effect of Water}

Generally speaking, when an object vibrates in the liquid, the resonant frequency is different from that in the air because it vibrates with near-area water. When installing a receiver in water and applying the supersonic of the $32 \mathrm{kHz}$, the authors have known the exact size of the receiver from former study. The receiver resonating in the water in this case, is shown in Fig. 5 and Table I.

The authors have made finite element model of the receiver and changed mass density from $10 \mathrm{~g} / \mathrm{cm}^{3}$ to $12 \mathrm{~g} / \mathrm{cm}^{3}$, and obtained eigenvalue at each case. When mass density is $11.24 \mathrm{~g} / \mathrm{cm}^{3}$, the natural frequency is coincidence with the frequency of the $32 \mathrm{kHz}$ in the experiment. It indicates that the mass density should be added by $3.21 \mathrm{~g} / \mathrm{cm}^{3}$ (mass density of SUS304 is $8.03 \mathrm{~g} / \mathrm{cm}^{3}$ ). In this research, 11.24 $\mathrm{g} / \mathrm{cm}^{3}$ is applied to finite element model as the mass density in water.

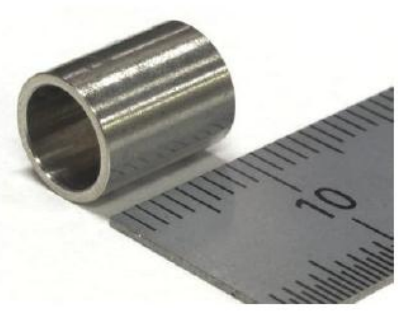

Fig. 5. Cylindrical type receiver.

\section{Frequency Response Analysis of the Cylinder Type Receiver}

The authors have made a dynamic model of the cylinder type receiver. The pressure load is added on the surface as acoustic pressure of finite element method. Mass density is $11.24 \mathrm{~g} / \mathrm{cm}^{3}$.

TABLE I: DIMENSIONS OF RECEIVER RESONATED IN WATER

\begin{tabular}{|c|c|c|}
\hline Length $[\mathrm{mm}]$ & $\begin{array}{c}\text { Outside diameter } \\
{[\mathrm{mm}]}\end{array}$ & Inside diameter $[\mathrm{mm}]$ \\
\hline 10.00 & 8.00 & 6.41 \\
\hline
\end{tabular}

The wavelength of the supersonic is longer enough than the size of the receiver, so the unit load $\left(1.0 \mathrm{~N} / \mathrm{mm}^{2}\right)$ added to a cylinder receiver is set.

A frequency response is analyzed by a frequency bandwidth of the surrounding $3 \%$ about 99 points in the case of resonant frequency $f_{r}=31997.15 \mathrm{~Hz}$ (median). The response is shown in Fig.6. It shows that the maximum amplitude of vibration $(2.57 \mathrm{x}$ is $10-7 \mathrm{~mm})$ is obtained in the resonant frequency $f_{r}=31997.15 \mathrm{~Hz}$. On the other hand, the amplitude of vibration $\left(1.39 \times 10^{-8} \mathrm{~mm}\right)$ is observed in frequency $31037.23 \mathrm{~Hz}$ at a negative side edge of the measuring range. So, the gain of the amplitude in non-resonance and resonant is 18.5. This is the effect of resonant phenomena.

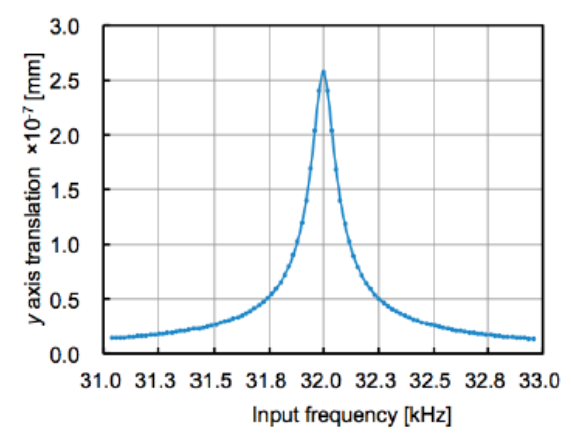

Fig. 6. Frequency response analysis.

Fig. 7 shows the relationship between the inside diameter of the receiver and resonant frequency by finite element method. As the diameter is larger, resonant frequency is decreasing linearly.

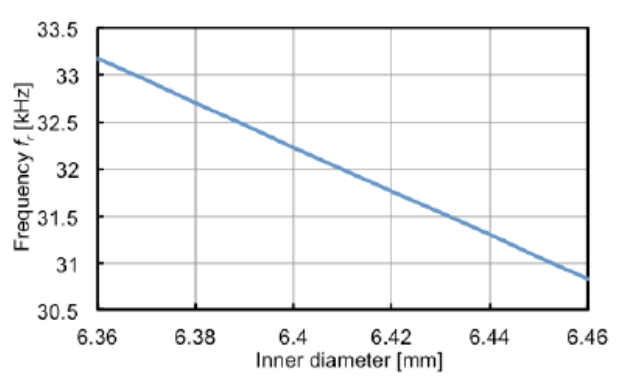

Fig. 7. Relationship of resonant frequency and inner diameter.

It is very difficult to coincide the frequencies of the vibrator and the receiver due to water effect and processing accuracy.

\section{IMPACT TYPE STENT MOTOR}

The authors have also designed a stent motor with a discus type receiver. Compering with the cylindrical type, it is easy to receive ultrasonic. The discus type receiver's thickness is $0.1 \mathrm{~mm}$ and the diameter is $11.0 \mathrm{~mm}$. The stator has coil 
shape; the diameter of the coil wire is $0.4 \mathrm{~mm}$, pitch $1.57 \mathrm{~mm}$, 7 turns, the outside diameter $11 \mathrm{~mm}$. The receiver is bent vertically and connected to the coil. It uses no resonant phenomenon. The finite element model is shown in Fig.8.

The sine wave pressure of $20 \mathrm{kHz}$ and $1.0 \mathrm{~N} / \mathrm{mm}^{2}$ modeled on acoustic pressure is applied to this model's receiver surface. The finite element model simulates time domain response. The interesting node point is Node $\mathrm{S}$ in Fig.8 (Node $\mathrm{S}=(0.0630684,5.499638,-7.199356))$. This point is located on the surface where it makes contacts with blood vessel. An analysis results are shown in Fig.9 with respect to the displacement of Node's small $x$ and $y$ direction. Applied vibration can be observed.

The wave along $\mathrm{x}$-axis and $\mathrm{y}$-axis draws sinusoidal curve with 129.8 degree phase difference. It indicates that the Node draws elliptical motion and this motor may work as an ultrasonic actuator.

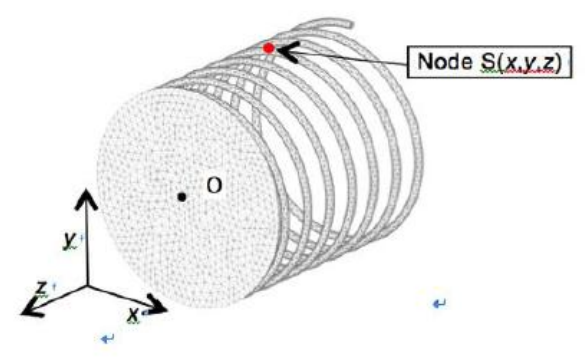

Fig. 8. FEM model.

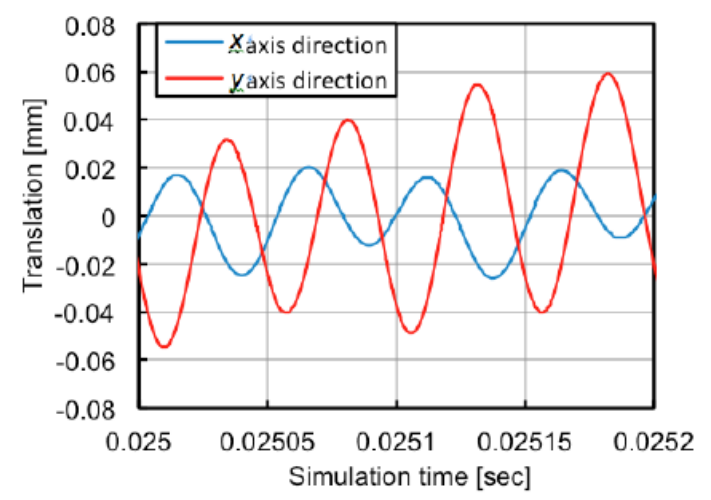

Fig. 9. Vibration in $x$ axis and $y$ axis.

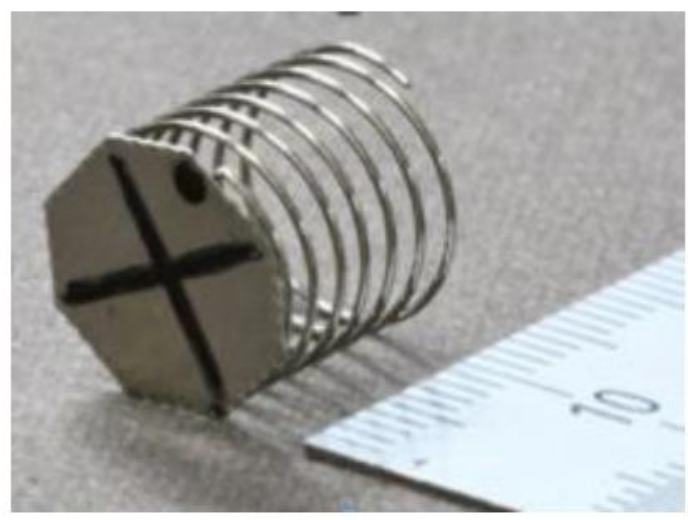

Fig. 10. Discuc type stent motor.

\section{A. Experiments of Discus Type Stent Motor}

The authors have made experiments in water with the discus type stent motor shown in Fig.10. The motor is made of SUS304 and the receiver (diameter $4.8 \mathrm{~mm}$ ) and the coil (20.0mm length) are connected by solder. As a blood vessel model, MC nylon block with nut shape through hole in the center is prepared. The overview is shown in Fig.11. The authors have controlled output wattage of Langevin vibrator to drive it in water. The rotational speed of it is shown in Fig.12. It is rotating with rate of $5.28 \mathrm{rpm}$ and $4.19 \mathrm{rpm}$ in the case of irradiating $70 \mathrm{~W}$ and $35 \mathrm{~W}$, respectively.

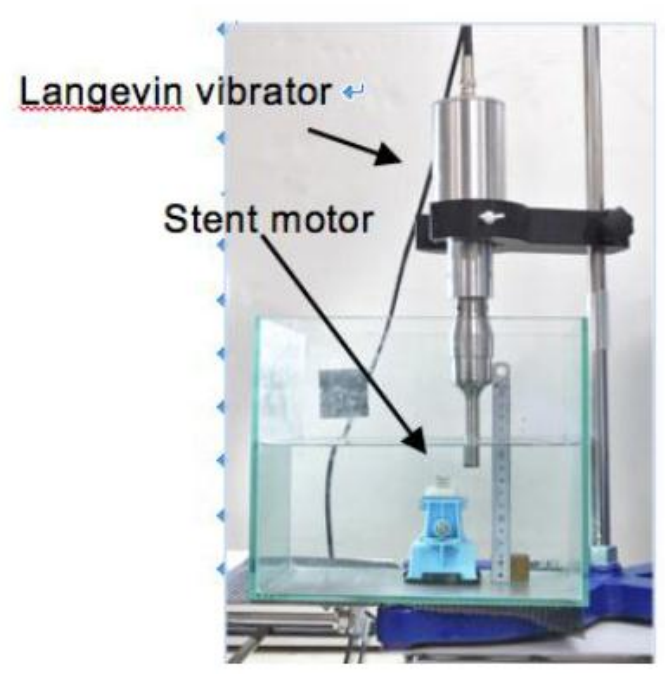

Fig. 11. Experimental apparatus.

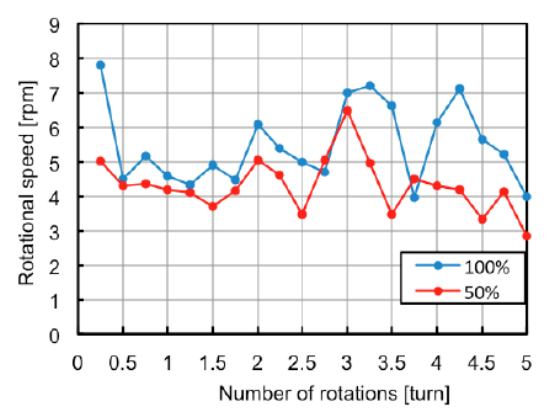

Fig. 12. Rotational speed in water.

\section{CONCLUSIONS}

The authors have concluded as follows.

1) The stent motor using resonant frequency requires high accuracy in design and processing. The accurate stent motor has good resonant characteristics in water. It shows high potential about use in water.

2) The stent motor using impact is ease to use in water. It shows good maneuverability in water.

\section{REFERENCES}

[1] S. Chen, B. Mulgrew, and P. M. Grant, "A clustering technique for digital communications channel equalization using radial basis function networks," IEEE Trans. on Neural Networks, vol. 4, pp. 570-578, July 1993.

[2] K. Masamune et al., "Development of an MRI compatible needle insertion manipulator for stereotactic neurosurgery," Journal Image Guided Surgery, vol. 1, 242 -247, 1995.

[3] T. Mashimo and S. Toyama, "Development of the translational and rotational piezoelectric actuator using a single stator $\left(1^{\text {st }}\right.$ report)-Design of the stator by finite element method analysis," Journal of the Japan Society for Precision Engineering, vol.74, No.3, 292-297, 2008.

[4] P. A. M. Ferreira, "High-performance load-adaptive speed control for ultrasonic motors," IFAC, J.Control Engineering Practice, vol. 6, no. 1, 1-10, 1998. 
[5] M. Hoshina, T. Mashimo, N. Fukaya, O. Matsubara, and S. Toyama, "Spherical ultrasonic motor drive system for pipe inspection," Advanced Robotics, vol. 27, 2013.

[6] T. Mashimo and S. Toyama, "Vibration analysis of cubic rotary-linear piezoelectric actuator," IEEE Transactions on Ultrasonics, Ferroelectrics, and Frequency Control, vol. 58, 844-847, 2011.

[7] T. Mashimo, S. Toyama, and H. Ishida, "Design and implementation of a spherical ultrasonic motor," IEEE Transactions on Ultrasonics, Ferroelectrics, and Frequency Control, vol. 56, 2514-2521, 2009.

Shigeki Toyama received Ph.D. degree in engineering from University of Tokyo, Tokyo, Japan, in 1981. Now he works at Tokyo University of Agriculture and Technology. His current research interests include robotics, actuator, and space and deepsea technology.

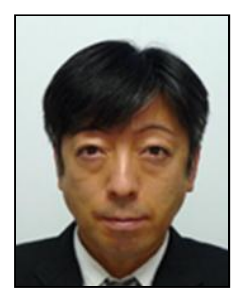

Uichi Nishizawa received Ph.D. degree in engineering from Tokyo University of Agriculture and Technology, Tokyo, Japan, in 2008. Now he works at Tokyo University of Agriculture and Technology. His current research interests include robotics, aerospace engineering, and welfare technology.

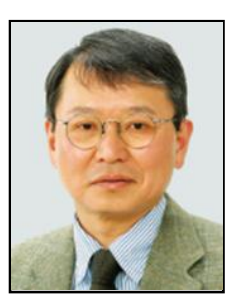

